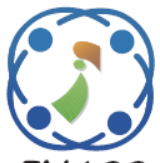

\title{
A Robust Estimation and Tracking of Channel for Time and Frequency Selective MIMO-OFDM Systems
}

\author{
Gadda Archana Devi ${ }^{1 *}$ \\ Moparthy Gurunadha Babu² \\ ${ }^{1}$ Shri Jagdishprasad Jhabarmal Tibrewala University, Rajasthan, India \\ ${ }^{2}$ Department of Electronics and Communication Engineering, \\ CMR Institute of Technology, Hyderabad, Telangana, India \\ * Corresponding author's Email: garchanadevi25@gmail.com
}

\begin{abstract}
Due to the higher data transmission networks and maximum efficiency, multiple-input-multiple-output orthogonal frequency division multiplexing (MIMO-OFDM) has become most vital wireless communications system in nowadays. Accurate estimation of channel under severe time and frequency selective fading channels has been required by these MIMO-OFDM systems for enabling high practical mobility. Established methods trust on nonrealistic estimations or result in impractical complexities. In this, we presented a new MIMO-OFDM channel estimation (CE) scheme for pilot length minimizing by designing novel pilot bits from the Hadamard matrices of Paley (HM-P) type I allows its orthogonal and diagonal constant matrix structures manipulations. Additionally, we also implemented a method which estimates the channel impulse response length to enamour existed number of multipath within the channel by stretching the length of pilot. Furthermore, our proposed channel estimation scheme modified to derive the initial fluctuation of channel and its Doppler rate, afterwards during the payload applied for tracking of MIMO channel using fast adaptive hybrid linear quadratic estimation (FAH-LQE) filter. Experimental analysis has done in MATLAB environment under Rayleigh distribution and achieved excellent channel estimation and channel tracking performances over the state-of-art channel estimation algorithms. Our proposed scheme also achieved lower diversity of transmitting bits by applying FAH-LQE filter and the superior estimation of channel under multipath conditions while increasing the number of pilot bits sent per transmitting antenna.
\end{abstract}

Keywords: MIMO-OFDM, Channel estimation, Channel tracking, Diversity, Paley-Hadamard matrix, Channel impulse response (CIR), Channel frequency response (CFR), Kalman filter and FAH-LQE.

\section{Introduction}

In orthogonal frequency division multiplexing (OFDM) systems, a frequency selective (FS) channel is commuted into an accumulation of flat fading channels, which can be indemnified by simply using an equalizer of one-tap [1]. Therefore, OFDM can greatly alter the design of equalizer while enabling rather higher data rates, which leads that it is used as a vital physical layer in many commercial systems. OFDM has been expanded further to Multiple-input multiple-output (MIMO), which is known as MIMO-OFDM, an extension for the general OFDM system. It's a modish technology in the era of wireless communications. MIMO-
OFDM provides higher mobility, high robustness versus multipath fading and has capability of high rate transmission. The higher requirements for improved reliability and high data rates in today's wireless communication have made multiple input multiple-output (MIMO) techniques as one of the key solutions for future generation wireless communication e.g. wireless highways [2] due to its potentiality to multipath exploitation while actualizing enhanced performance in spatial multiplexing, array gain and power. To obtain higher data rates, MIMO systems utilize ' $\mathrm{N}$ ' number of transmitting and receiving antennas. In case of fast time varying MIMO systems, channel equalization has to be done effectively to remove Inter Symbol Interference (ISI) which usually occurs 
in frequency selective channels, so OFDM is used for this purpose. Due to the integration of OFDM technologies, MIMO-OFDM systems can boost the rate of data transmission while dealing frequencyselective (FS) fading [3, 4]. By concurrently exploiting the time, space and frequency domains, MIMO-OFDM also has substantial possible efficient performances over conventional wireless technologies.

It also has a capability of gratifying the nextgeneration wireless networks requirements such as Wireless Local Area Networks (WLANs), Wireless Fidelity (Wi-Fi), and World-wide interoperability for Microwave Access (Wi-MAX), Cognitive Radio, 3rd Generation Partnership Project (3GPP) and LTE. However, these sorts of systems should be capable to gain exact channel information to grant and enable high mobility practicability and for recovery of symbols in high speed while handling co-channel interference (CCI) and time selective (TS) fading.

This article proposes a robust MIMO-CE-CT schemes which have been invented to actualize practical communications in both the channels those are TS and FS. Our method is also vital for the consequent $\mathrm{CT}$ provision for individual CFR or CIR to receiver's resiliency assurance versus the environment of severe TS fading and accurate receiver's setting attainment during the recovery of symbol with the process of decision feedback. The MIMO-OFDM system is chosen here to demonstrate the effectiveness and robustness of our proposed CE and CT methods, while their enhancements to any further MIMO-based communication systems are straightforward.

Rest of the paper as follows: section 2 describes the related work already done in the field of channel estimation, section 3 explains the description of problem, system model has been described in section 4, simulation analysis in section 5 followed by conclusion in section 6 with references.

\section{Related work}

Various early attempts have successfully made for estimating the channel in communication systems such as code division multiplexing (CDMA) and OFDM systems [5, 6]. Due to the computational onus of Eigen value decomposition (EVD) and ineptness for adaptive processing of state-of-art schemes in the literature like multiple signal classification (MUSIC) method [7] and estimation of signal parameters via rotational invariance techniques (ESPRIT) [8], authors in [9, 10] proposed a low-complexity subspace tracking algorithms to track the subspace of signal recursively. Channel estimation in MIMO OFDM system for fast time varying channel is already been proposed using Extended Kalman Filter (EKF) but that process uses pilot symbols to train the filter, which degrades the data throughput by occupying the available bandwidth for data transmission [11, 12]. Moreover, EKF need to compute Jacobians to linearize the nonlinear channel estimation. Most of the CE schemes are being done in frequency domain, where the parameters to be estimated are huge, when compared to estimation in time domain, where only some limited parameters about the channel is to be estimated [13, 14]. Generally, pilot-aided (PA) $\mathrm{CE}$ algorithms have been adopted for established MIMO-OFDM systems, where non-parametric imminent can be attained by exploiting the channel frequency response (CFR)'s time and frequency correlation [15]. Without any restraints about the channel, the estimated trouble's dimension can be quite huge. Still, in wireless communication systems the radio channel is frequently qualified by a few paths those are prevalent, typically two to six [16]. Moreover, the transmission of data at higher speeds results in a thin multipath channel. The signal subspace attribute of the correlation matrix can be effectively mitigated when the construction of correlation matrix of a channel based on the parametric channel model [16]. Although the approaches with orthogonal pilot [15-17] can eliminate pilot interference, the pilot overhead issue has not been taken into account, which is one of important issues in massive MIMO-OFDM.

Several smart CE and channel tracking (CT) schemes [18-20] have been proposed to furnish estimates on the channel impulse response (CIR) or CFR. Recently, to take on the issue of the pilot contamination problem, a substantial research work had been presented in the literature. A Bayesian estimator was adapted for a multi-cell scenario, to the interference suppression from users when certain non-overlapping considerations satisfied by the channel covariance matrices on their prevalent subspace [21]. Since unlike users channels incline to be a pair wise orthogonal in a massive antenna system, blind discrimination is accomplishable between the interferences and desired signals based on the average channel gains which are prior known [22]. Recently, author in [23] proposed an algorithm for $\mathrm{CE}$ by path diversity exploitation in both domains i.e., angle and power. In general, the trend of adopting or trusting on the prior knowledge of a) the CIR length and b) Doppler rate, often become the constriction to trust on sufficiently higher order polynomial model leading in complicated algorithms, showing an impractical solvent for real 
time $\mathrm{CE}$ where the pilot optimality finally reckons on the CE speed. To overcome all the abovementioned issues and to improve the robustness and reliability of CE algorithms in MIMO-OFDM systems, this article proposes a robust MIMO-CECT schemes which have been invented to actualize practical communications in both the channels those are TS and FS. Our method is also vital for the consequent $\mathrm{CT}$ provision for individual CFR or CIR to receiver's resiliency assurance versus the environment of severe TS fading and accurate receiver's setting attainment during the recovery of symbol with the process of decision feedback. The MIMO-OFDM system is chosen here to demonstrate the effectiveness and robustness of our proposed $\mathrm{CE}$ and $\mathrm{CT}$ methods, while their enhancements to any further MIMO-based communication systems are straightforward.

\section{Problem description}

An issue observed in the communication system is diversity in the transmitted signal over the channel. The diversity is observed over time and frequency for the transmitting bits. Though, the usage of multiple antenna models in communication provides a higher throughput, the usage of multiple antenna arrays and frequency division in communication results in higher diversity of transmitting bits. Hence, the Kalman filter at the estimator unit takes a longer time of convergence, resulting in slower processing. The issue of mobility is observed in all the existing communication system. In the case of communication, due to multiple antennas the transmitting signals are propagated in different allocated power and frequency. These signals are transmitted over the channel, which travels through multiple channels scattered via different medium, and obstacle encountered. The signal scattering results in multi-pathing and the received signals are observed at lower strength. Though Kalman filtration for different fading issues are been developed, it need to improve to encounter the issue of variation in fading within each communication period, which varies due to user mobility. The encountered fading in such case is dynamic, as with the mobility of user the communicating channel keeps varying with time. With these objectives in this work, a fast-adaptive hybrid Kalman filtration in consideration to channel diversity and mobility concern is focused. The objective of solving the divergence problem more effectively and optimal state information estimation has been focused by implementing the channel estimation and channel tracking algorithms.

\section{System model}

Here, we had considered severe TS and FS fading channels and simulated using the Jakes model [33]. The time domain (TD) time-varying sampled CIR from $m^{\text {th }}-T_{x}$ antenna to $n^{\text {th }}-R_{x}$ antenna can be termed as

$$
\underset{1, t)]^{T}}{\mathbb{h}_{n m}(t)=\left[h_{n m}(0, t)\right.} h_{n m}(1, t) \cdots \quad h_{n m}(L-
$$

where $L$ denotes the number of multipath on each $T_{x}-R_{x}$ antenna pair and $h_{n m}(\ell, t)$ is the individual channel coefficient of TD on $\ell$ path at time $t$. The MIMO channel will be formed by $\mathbb{h}_{n m}(t)$ shown in (4) later. The accompanying time-varying sampled CFR from $m^{t h}-T_{x}$ antenna to $n^{t h}-R_{x}$ antenna can be found by applying the inverse fast-Fourier transform function to $\mathbb{h}_{n m}(t)$ in (1) as:

$$
\begin{gathered}
g_{n m}(t)=\operatorname{ifft}\left(\mathbb{h}_{n m}(t), N\right)= \\
{\left[g_{n m}(0, t) \quad g_{n m}(1, t) \cdots \quad g_{n m}(N-1, t)\right]^{T}}
\end{gathered}
$$

where $N$ is the number of ifft point and $g_{n m}(n, t)$ is the channel coefficient of individual frequency domain (FD) on $n^{\text {th }}$ subcarrier at time $t$. The Doppler rate $f_{D} T$ (where $\mathrm{f}_{\mathrm{D}}$ denotes maximum Doppler frequency and $T$ is the baud duration) is used to regularize the time-correlation between the contiguous TD coefficients as:

$E\left\{h_{n m}\left(t_{1}\right) h^{*}{ }_{n m}\left(t_{2}\right)\right\} \approx J_{0}\left(2 \pi f_{D} T\left|t_{1}-t_{2}\right|\right)$

Where $J_{0}(\cdot)$ denotes the first kind's Bessel function with zero-order. Thus, the resultant $g_{n m}(n, t)$, in (2) must have the similar properties of time varying with the equal Doppler rate and the time-correlation between the contiguous FD coefficients as $h_{n m}(\ell, t)$. Our proposed MIMO-CE-CT scheme demonstrated in Figs. 1 and 2 to estimate the corresponding CIRs and its ' $L$ ' length while exposing the channel variation information via an efficient enlargement of pilot (EEP) scheme.

\subsection{MIMO-CE utilizing HM-P codes}

To provide a virtual 'division' on individual CIR in FS case, each basic channel matrix consisting of all CIRs, $\mathbb{h}_{n m}(t)$ with respect to each $T_{x}$ antenna can be written as: 


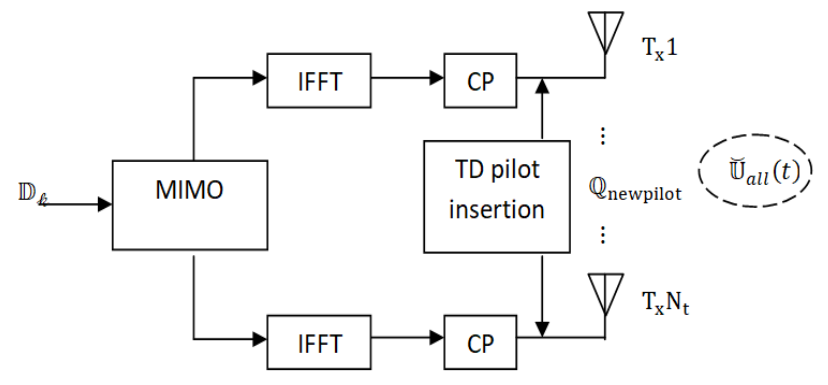

Figure. 1 Transmitter block diagram for proposed EEP$\mathrm{CE}$ and FAH-LQE-CT

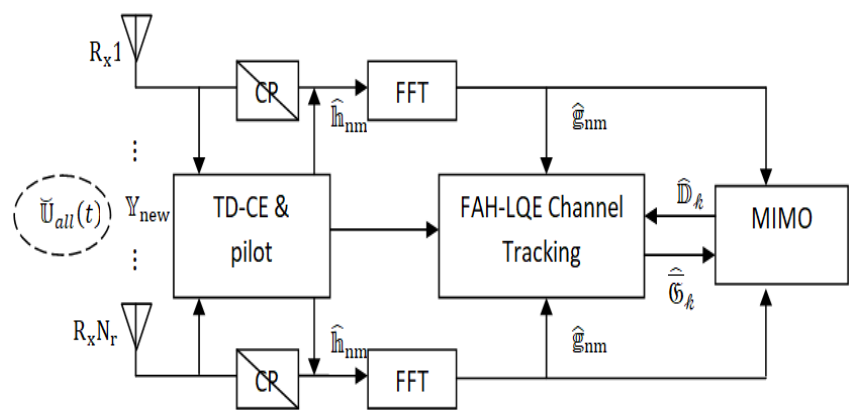

Figure. 2 Receiver block diagram for proposed EEP-CE and FAH-LQE-CT

$$
\begin{gathered}
\mathbb{U}_{m}(t)=\left[\begin{array}{c}
\mathfrak{h}_{1 m}{ }^{T}(t) \\
\vdots \\
\mathbb{h}_{N, m}{ }^{T}(t)
\end{array}\right]= \\
{\left[\begin{array}{ccc}
h_{1 m}(0, t) & \cdots & h_{1 m}(L-1, t) \\
\vdots & \ddots & \vdots \\
h_{N, m}(0, t) & \cdots & h_{N, m}(L-1, t)
\end{array}\right]}
\end{gathered}
$$

Where $m=1$ to $N_{t}$ and number of multipath exists in each CIR is denoted by $\mathrm{L}$.

Further concatenate all $\mathbb{U}_{m}(t)\left(m=1, \cdots, N_{t}\right)$ to alleviate an efficient MIMO-CE to form the complete MIMO channel as:

$$
\mathbb{U}_{\text {all }}(t)=\left[\begin{array}{lll}
\mathbb{U}_{1}(t) & \cdots & \mathbb{U}_{N_{t}}(t)
\end{array}\right]
$$

Temporarily, in order to distinguish the 'virtual variation' the time-index ' $t$ ' has been changed to ' $n$ ' due to ' $n$ ' pilot sequence bits as oppose to the genuine variation in time. Therefore, interactions in TD signal at $n$th sample at the receiver can be viewed as:

$$
\begin{aligned}
& \mathbb{y}(n)=\mathbb{U}_{\text {all }}(n) \mathfrak{I}(n)=\left[y_{1}(n) \cdots y_{N_{r}}(n)\right]^{T} \\
& \mathfrak{I}(n)=\left[\mathbb{q}_{1}(n) \cdots \mathbb{M}_{N_{t}}(n)\right]^{T} \\
& \mathbb{q}_{m}(n)=\left[q_{m}(n) q_{m}(n-1) \cdots q_{m}(n-L+1)\right]^{T}
\end{aligned}
$$

Note that $\mathfrak{I}(n)$ consists of $N_{t}$ number of $T_{x}$ vector, $\mathbb{q} m(n)$ sent by individual $m^{t h}-T_{x}$ antenna. Each $q_{m}(n)$ contains $L$ length of $T_{x}$ pilot bits; from current $\mathrm{n}^{\text {th }}$ bit to past $(n-L+1)^{t h}$ bit, which convolve with different coefficients $\mathbb{h}_{n m}(\ell, t)$. The aim is to design pilot codes in $\mathbb{q}_{m}(n)$ such that pilot vectors $\mathfrak{J}(n)$ can differentiate individual $\mathrm{T}_{\mathrm{x}}$ antenna for practical separatism of all its CIRs. The pilot matrix $\mathbb{Q}_{\text {pilot }}$ can be formed as:

$$
\mathbb{Q}_{\text {pilot }}=[\mathfrak{I}(n) \mathfrak{I}(n+1) \cdots \mathfrak{I}(n+r-1)]
$$

where the order of chosen matrix is denoted with $r$. Each row sequence in $\mathbb{Q}_{\text {pilot }}$ is designed to be orthogonal w.r.t other row sequences. Next, the CE can be performed with ' $L$ ' known a priory as:

$$
\widehat{\mathbb{U}}_{\text {all }}=\mathbb{Y} \mathbb{Q}^{H} \text { pilot }
$$

Where the corresponding $R_{x}$ pilot matrix has denoted by $\mathbb{Y}$ with the proper $\mathbb{y}(n)$ samples collection at every ' $L$ ' intervals as:

$$
\mathbb{Y}=[\mathbb{y}(n) \mathbb{y}(n+L) \cdots \mathbb{y}(n+(r-1) L)]
$$

Our main challenge is to design (9) in such a way that the gap between the $y(n)$ collection intervals in $\mathbb{Y}$ can be further mitigated to include contiguous samples, while assuring the accuracy of CE in (10), which can be accomplished by utilizing the Hadamard matrices of Paley type I which provides both orthogonal and diagonal constant matrix structures of unique combined. Our analysis from Eq. (4) to (11) exposes that the diagonal constant matrix structure can be exploited such that only $r+$ $2(L-1)$ number of pilot bits (instead of $r L$ ) is required per $\mathrm{T}_{\mathrm{x}}$ antenna. Pilot bits those not in the region of $r+2(L-1)$ can avoid being sent, while keeping the exact $\mathbb{Q}_{\text {pilot }}$ formation at the receiver for CE in Eq. (10). This appropriates that almost every successive $\mathbb{y}(n)$ samples to be utilized right away without any gap, instead of waiting every ' $L$ ' interval as in Eq. (11), thereby further expurgating the length of pilot and overall processing time of CE that is crucial in the TS channels case.

The MIMO-CE approach is further implemented to a) approximate the CIRs length and b) disclose the variation of channel. In reality, length ' $L$ ' in each CIR is unknown, while the ultimate size of $\widehat{\mathbb{U}}_{\text {all }}$ in Eq. (10) depends purely on the collected samples $\mathbb{y}(n)$ in $\mathbb{Y}$ and corresponding $\mathbb{Q}_{\text {pilot }}$ formation at the receiver. Hence, it is vital to allow flexible enlargement in the length of pilot to provide every possible ' $L$ ' while restraining the MIMO-CE process integral. To effectively 'capture' this 
information, an approximation EEP model of $\mathbb{U}_{m}(t)$ in (4) can be formed as:

$$
\begin{gathered}
\breve{\mathbb{U}}_{m}(t)=\left[\begin{array}{c}
\breve{\mathbb{h}}_{1 m}{ }^{T}(t) \\
\vdots \\
\breve{h}_{N, m}{ }^{T}(t)
\end{array}\right]= \\
{\left[\begin{array}{ccc}
\breve{h}_{1 m}(0, t) & \cdots & \breve{h}_{1 m}\left(L_{e s t}-1, t\right) \\
\vdots & \ddots & \vdots \\
\breve{h}_{N, m}(0, t) & \cdots & \breve{h}_{N, m}\left(L_{\text {est }}-1, t\right)
\end{array}\right]}
\end{gathered}
$$

Where $\mathrm{L}_{\text {est }}$ is the estimated CIR length and $\mathrm{L}_{\mathrm{est}}>=\mathrm{L}$ so that estimated $\widetilde{\mathbb{U}}_{m}(t)$ in Eq. (12) is always wider than $\mathbb{U}_{m}(t)$ in Eq. (4), intuitively, sending a longer pilot sequence of pilot vectors will ensure that the estimate of $\widetilde{\mathbb{h}}_{n m}{ }^{T}(t)$ is always sufficient to approximate the actual $\mathbb{h}_{n m}(t)$, with additional (Lest-L) CIR 'residues' at the end of $\breve{h}_{n m}{ }^{T}(t)$ estimate. These residues are usually near 'zero' value, which can be removed (or further utilized). However, using only a single $\widetilde{\mathbb{U}}_{m}(t)$ EEP model in Eq. (12) can render insufficient CE accuracy in the high noise case. Thus, to guarantee a better expectation of $\mathrm{CE}$, a further extended approximation on EEP model on $\mathbb{U}_{\text {all }}(t)$ is required as

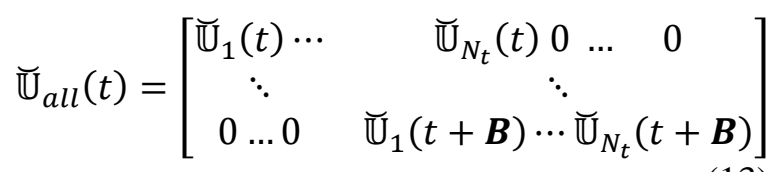

Where $\widetilde{\mathbb{U}}_{\text {all }}(t)$ contains $(\mathbf{B}+1)$ blocks of $\widetilde{\mathbb{U}}_{m}(t)$ in succession which implies they can now be jointly evaluated to increase the CE accuracy. For a timeinvariant channel, all $\widetilde{\mathbb{U}}_{m}(t)$ to $\widetilde{\mathbb{U}}_{m}(t+\boldsymbol{B})$ will be quite similar, thus, render a positive estimation of $\widetilde{U}_{m}(t)$. When the channel is time-varying, changes in CIRs are also gradually revealed across from all $\widetilde{\mathbb{U}}_{m}(t)$ to $\widetilde{\mathbb{U}}_{m}(t+\boldsymbol{B})$, providing the vital clue to unveil the time-variation information (i.e. the Doppler rates and travel velocities) while the basic $\mathrm{CE}$ is carried out. In fact, $L_{\text {est }}$ can initially take a larger value to include $\boldsymbol{B}$ in order to estimate $\widetilde{\mathbb{U}}_{m}(t)$ and the length of CIR in Eq. (12) first (as the resultant residues will implicate the actual CIR length) and then adapt $\boldsymbol{B}$ as $\left(\mathrm{L}_{\mathrm{est}}-L\right)$ to further process $\widetilde{\mathbb{U}}_{\text {all }}(t)$ in Eq. (13). To obtain this, $\mathbb{Q}_{\text {pilot }}$ in Eq. (9) must be capable to be plaint flourished to gratify the $\widetilde{\mathbb{U}}_{\text {all }}(t)$ formation in Eq. (13), The key highlight in the proposed MIMO-CE is, while retaining the length of pilot to minimum requirement and resolving CCI, TS and FS channels concurrently for a massive MIMO channel during
CE. To demonstrate this, pilot bits in $\mathbb{q}_{m}(n)$ in Eq. (8) are elaborated to inject $\left(\boldsymbol{B}+L_{e s t}-L\right)$ additional bit per transmitting antenna as:

$$
\begin{aligned}
& \widetilde{q}_{m}(n)=\left[q _ { m } ( n ) q _ { m } ( n - 1 ) \cdots q _ { m } \left(n-L_{e s t}-\right.\right. \\
& \quad \boldsymbol{B}-1)]^{T}
\end{aligned}
$$

This will return a corresponding $\mathfrak{I}_{\text {new }}(n)$ and $\mathbb{B}(n)$ as:

$$
\mathbb{B}(n)=\breve{\mathbb{U}}_{\text {all }}(t) \widetilde{I}_{\text {new }}(n)=[\mathbb{y}(n+\boldsymbol{B}) \ldots \mathbb{y}(n)]^{T}
$$

Where $\mathbb{B}(n)$ contains few base vector $\mathbb{y}(n)$ in Eq. (6) in succession. $\mathfrak{J}_{\text {new }}(n)$ can still be formulated using Eq. (7) with $\widetilde{q}_{m}(n)$ in Eq. (14) by replacing $q_{m}(n)$ in Eq. (8). Therefore, the new pilot matrix $\mathbb{Q}_{\text {newpilot }}$ expanded from Eq. (9) with a new Hadamard order- $r$ :

$$
r>N_{t} \times\left(L_{e s t}+\boldsymbol{B}\right)
$$

Therefore, the new CE with efficient enlargement of pilot bits of $\boldsymbol{r}+2\left(L_{e s t}+\boldsymbol{B}-1\right)$ per $T_{x}$ antenna and is performed as:

$$
\widehat{\mathbb{U}}_{\text {all }}=\mathbb{Y}_{\text {new }} \mathbb{Q}_{\text {newpilot }}^{H}
$$

Where the corresponding new $R_{x}$ pilot matrix denoted as $\mathbb{Y}_{\text {new }}$ with the $\mathbb{B}(n)$ proper collection of blocks from Eq. (15) as:

$$
\begin{gathered}
\mathbb{Y}_{n e w}=[\mathbb{B}(n) \mathbb{B}(n+1) \cdots \mathbb{B}(n+r-2) \mathbb{B}(n+ \\
\left.\left.r+L_{\text {est }}+\boldsymbol{B}-1\right)\right]
\end{gathered}
$$

Note that there is a tiny waiting gap between the two blocks $\mathbb{B}(n+r-2)$ and $\mathbb{B}\left(n+r+L_{\text {est }}+\boldsymbol{B}-\right.$ 1)to adjust HM-P formation in $\mathbb{Q}_{\text {newpilot }}$ method in Eq. (17). Once $\widehat{\mathbb{U}}_{\text {all }}(t)$ is obtained, each $\widetilde{\mathbb{U}}_{m}(t)$ within can be measured jointly for the channel time-variation information and its ' $L$ ' length in CIRs

\subsection{Kalman channel tracking}

To accompaniment our proposed CE-EEP method in the case of TS channel, the Kalman approach [24] was utilized for tracking the channel. $\mathrm{TD} \widehat{\mathbb{R}}_{n m}(t)$ are changed into FD $\widehat{g}_{n m}(t)$ and rearranged as $\overline{\mathfrak{G}}_{k}$, and tracked subsequently by the Kalman algorithm below:

Step 1: Predicting Equations 


$$
\begin{aligned}
\widehat{\widehat{\mathfrak{G}}}^{-}{ }_{k+1}{ }^{T} & =\mathbb{A} \widehat{\overline{\mathfrak{G}}}_{k}{ }^{T} \\
\mathbb{P}^{-}{ }_{k+1}^{T} & =\mathbb{A} \mathbb{P}_{k} \mathbb{A}^{T}+Q
\end{aligned}
$$

\section{Step 2: Measurement equations}

$$
\begin{aligned}
& \mathbb{K}_{k}=\mathbb{P}_{k}^{-}\left(\widehat{\mathbb{D}}_{k}^{T}\right)^{H}\left(\widehat{\mathbb{D}}_{k}^{T} \mathbb{P}_{k}^{-}\left(\widehat{\mathbb{D}}_{k}^{T}\right)^{H}+\sigma^{2}\right)^{-1} \\
& \widehat{\widehat{\mathfrak{G}}}_{k}{ }^{T}=\widehat{\widehat{\mathfrak{G}}}_{k}{ }^{T}+\mathbb{K}_{k}\left(\mathbb{X}_{k}^{T}-\widehat{\mathbb{D}}_{k}^{T} \widehat{\widehat{\mathfrak{G}}}_{k}{ }^{T}\right) \\
& \mathbb{P}_{h}=\left(I_{N_{t} N}-\mathbb{K}_{h} \widehat{\mathbb{D}}_{h}^{T}\right) \mathbb{P}_{h}^{-}
\end{aligned}
$$

Where the Kalman gain is denoted as $\mathbb{K}_{k}$. $\mathbb{P}_{k}$ and $\mathbb{P}_{k}{ }^{-}$are the posterior and prior estimate error covariance matrices, respectively, $I_{N_{t} N}$ is the $N_{t} N$ size identity matrix, $Q$ and $\sigma^{2}$ are noise covariance for the process and measurement models. The channel transition matrix is denoted as $\mathbb{A}$. To afford an excellent CT mechanism, all the above parameters are tuned properly, refer to [24] therein for further insight.

\subsection{FAH-LQE channel tracking}

Discussion given in above section exploits about Kalman channel tracking, in which the proposed MIMO-CE has been complemented with CT mechanism. But it has drawback of adaptability of various changes in noise co-variance in Eq. (20) due to the surrounding environment. Hence, it is required to invariably update the noise estimation. So, we can get a more accurate expression of noise to further enhancement of CT mechanism more effectively. Here, we proposed an improvement for Kalman filter algorithm, in which it can accommodate to any small varies in channel environmental noise and will become a fast-adaptive hybrid linear quadratic estimation (FAH-LQE) filtering algorithm for more efficient channel tracking. The key of this algorithm is that it can invariably update the background noise estimation. Therefore, to determine whether there is a noise or not, we can set a sensible threshold(T). FAH-LQE contains two steps:

a) Updating the co-variance of channel noise $Q_{v}(n)$

b) Updating the threshold(T).

\subsubsection{Updating the co-variance of environmental noise} by

$$
Q_{v}(n)=(1-d) \times Q_{v}(n)+d \times Q_{u}(n)
$$

where $d$ is the loss factor that can limit the filtering memory length and enhance the new measurements role under the current estimates. According to channel output formula, its formula is:

$$
d=\frac{1-b}{1-b^{t+1}}
$$

where $\mathrm{b}$ is constant value with a range from 0.95 to 0.99 . Prior the implementation of $Q_{v}(n)$, we will use the co-variance of the current frame $Q_{u}(n)$ to equate with the threshold $\mathbb{T}$ which has been updated in the former iteration. If $Q_{u}(n)$ is less than or equal to $\mathbb{T}$, the current frame can be regarded as noise, and then we will re-estimate the co-variance of noise.

\subsubsection{Updating the threshold by}

$$
\mathbb{T}=(1-d) \times \mathbb{T}+d \times Q_{u}(n)
$$

As mentioned in Eq. (3), the properties of timevariation in the coefficients of FD in CFRs and the channel coefficients of TD in CIRs are same, where the time-correlation between the samples those are contiguous adopt similar Doppler rate, $f_{D} T$. Hence, it is vital to reflect this information in $\mathbb{A}$ in order to track ahead the estimates accurately. The estimate of $\widetilde{\mathbb{U}}_{\text {all }}(t)$ offers some vital clues as the displacements of coefficients from $\widetilde{\mathbb{U}}_{m}(t)$ to $\widetilde{\mathbb{U}}_{m}(t+\boldsymbol{B})$ in Eq. (13) and can be used to predict the corresponding mobile velocity, $\hat{\mu}$ and the Doppler shift frequency $\widehat{f_{d}}$, (from the known $T_{x}$ bit rate, $T$ ). With these estimates, the variation pattern of each coefficient in CFR can be approximated: First, forming a virtual waveform as: $\cos \left(2 \pi \widehat{f_{d}} T+\theta_{n m}(\ell)\right)$ with the phasor $\theta_{n m}(\ell)$ defined for each ' $\ell$ ' path from $m^{\text {th }}$ $T_{x}$ antenna to $n^{\text {th }}-R_{x}$ antenna. Next, taking the difference between every adjacent sample-pair and normalize them. The result offers a weighted projection 'gradient' with the variant rate like Eq. (3) and can be combined with the actual variation magnitude of each CFR coefficient that has already been revealed in the changes from $\widetilde{\mathbb{U}}_{m}(t)$ to $\widetilde{\mathbb{U}}_{m}(t+\boldsymbol{B})$ in Eq. (13) for time variation on the channels.

\section{Simulation analysis}

The results of the proposed MIMO-CE with the novel $\mathrm{PE}$ technique are studied using the parameters set in Table 1 below and the mean Frobenius norm squared error (MSE) of the channel estimates as compared to the original channels. For consistency, the MSEs are taken at the mid-length of the total pilot bits sent per each $\mathrm{T}_{\mathrm{x}}$ antenna. 
Table 1. Simulation parameters

\begin{tabular}{|c|c|c|}
\hline Symbol & Parameter & Value \\
\hline$f_{c}$ & Carrier frequency & $2.1 \mathrm{GHz}$ \\
\hline$R$ & Data rate per $\mathrm{Tx}$ & $600 \mathrm{kbps}$ \\
\hline$\mu$ & $\begin{array}{l}\text { Vehicle mobile } \\
\text { velocity }\end{array}$ & $55 \mathrm{~km} / \mathrm{hr}$ and $115 \mathrm{~km} / \mathrm{hr}$ \\
\hline$L$ & Length of CIR & $\begin{array}{c}3 \text { (equally spaced by one } \\
\text { sample) }\end{array}$ \\
\hline$N$ & FFT/IFFT size & 512 \\
\hline$f_{l}$ & Frame length & 200 OFDM symbols \\
\hline$N_{r} \times N_{t}$ & $\begin{array}{c}\text { MIMO } \\
\text { configuration }\end{array}$ & $\begin{array}{c}(3 \times 2): 3 \mathrm{Tx} \& 2 \mathrm{Rx} \\
\text { antennas }\end{array}$ \\
\hline
\end{tabular}

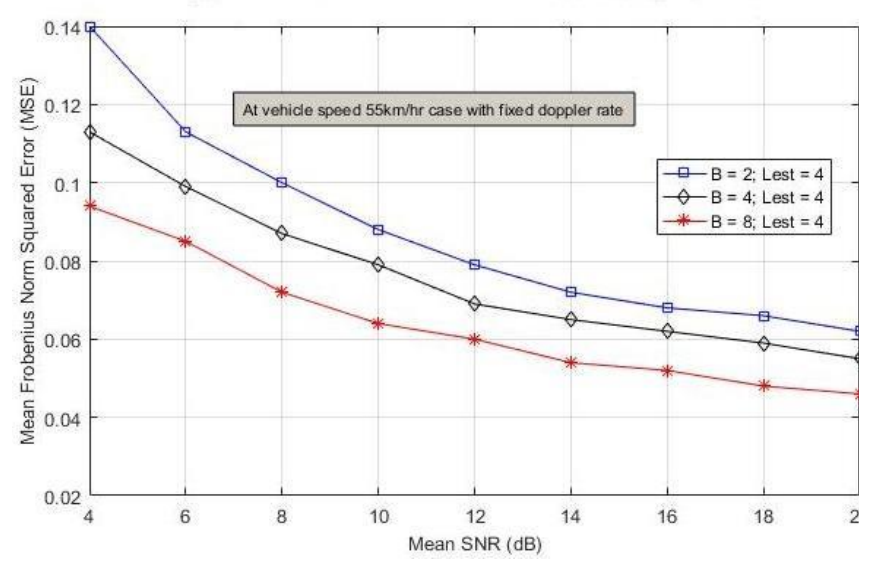

Figure. 5 Performance of EEP with different ' $\boldsymbol{B}$ ', for $55 \mathrm{~km} / \mathrm{hr}$

Table.2 Simulation Parameters

\begin{tabular}{|c|c|c|c|c|c|c|}
\hline $\boldsymbol{B}$ & 0 & 2 & 4 & 6 & 8 & 10 \\
\hline $\boldsymbol{r}$ & 8 & 12 & 20 & 20 & 24 & 32 \\
\hline Pilot bits sent per Tx & 12 & 20 & 32 & 36 & 44 & 56 \\
\hline
\end{tabular}

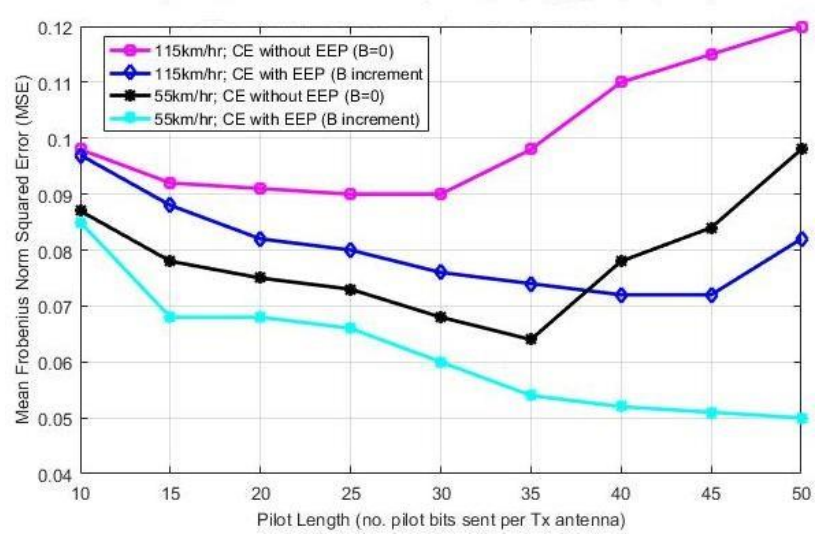

Figure. 6 Impact of CE with/without EEP for $\mu=55 \mathrm{~km} / \mathrm{hr}$ and $115 \mathrm{~km} / \mathrm{hr}$ channels

Fig. 5 shows the impact of the chosen ' $\boldsymbol{B}$ ' values of the EEP-CE technique on the estimation accuracy at different mean SNRs for a $\mu=55 \mathrm{~km} / \mathrm{hr}$ channel where the MSE improves as ' $\boldsymbol{B}$ ' increases for better averaging of channel estimates. The results suggested that using ' $\boldsymbol{B}$ ' blocks of $\breve{\mathbb{U}}_{m}(t)$ for joint evaluation, can help to further improve the channel estimates. However, as shown in Fig. 6 later as ' $\boldsymbol{B}$ ' becomes higher, the MSEs tends to depreciate due to the variation of the channel interaction with individual pilot bits. Fig. 6 compares the case using two channel velocities at $\mathrm{SNR}=15 \mathrm{~dB}$ and shows the impact of having proposed $\mathrm{CE}$ technique as the pilot length increases. For $(\boldsymbol{B}=0)$ case, where pilot length is allowed to increase without the help of proposed $\mathrm{CE}$, the result seems to suggest that there is an optimal pilot length for different channel speeds that limits the estimates to be further improved. With the use of proposed MIMO-CE ( $\boldsymbol{B}$ increment), the CE can cope better with the channel variations as MSEs have been improved as compared to the case without the use of PE technique. Fig. 6 is based on $L_{e s t}=$ $L=3$. For CE with the EEP assistance, the total pilot length: $r+2\left(L_{e s t}+\boldsymbol{B}-1\right)$ required per $\mathrm{T}_{\mathrm{x}}$ antenna (as the valid matrix order- $r$ in Eq. (16) and block ' $\boldsymbol{B}$ ' of EEP increases) is given in Table II. There is an optimal pilot length for different channel speeds that limits the estimates to be further improved.

Next, the behaviour of the CE and CT is jointly studied where CE pilot training is carried out for every data frame followed by the Kalman-CT and FAH-LQE-CT for each OFDM symbol delivery within the frame. The bit error rate (BER) system performance of a (3×2) MIMO-OFDM system under both TS and FS fading channels is shown in fig. 7 and fig. 8 for $115 \mathrm{~km} / \mathrm{hr}$ and $55 \mathrm{~km} / \mathrm{hr}$ respectively. Finally, the behaviour of the proposed CE and CT is jointly studied where $\mathrm{CE}$ pilot training is carried out for every data frame followed by the FAH-LQE-CT for each OFDM symbol delivery within the frame. We can observe that the values of BER get reduced while increasing the SNR values. The work presented in [25], shown that the analysis of both least square (LS) and minimum mean square error (MMSE) channel estimation algorithms. When SNR is $12 \mathrm{~dB}$ the LS estimator has a BER of $10^{-1}$, which is higher than that of the MMSE estimator. In addition, the MMSE estimator can achieve the same BER of $10^{-1}$ but at lower SNR of $8 \mathrm{~dB}$.

Thus, the higher the SNR, the better the MMSE estimator performs in comparison to the LS estimator. The CE algorithms proposed in [26] shown that the robustness of transformations-based CE approaches such as discrete Fourier transform (DFT), discrete cosine transform (DCT) under various channel distributions. 


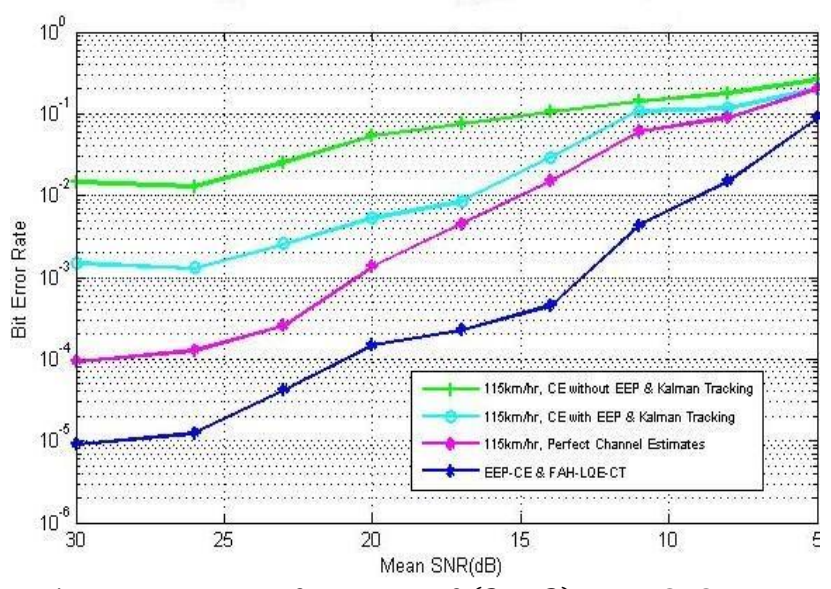

Figure. 7 BER performance of $(3 \times 2)$ MIMO-OFDM system with $\mu=115 \mathrm{~km} / \mathrm{hr}$ channels and $R=600 \mathrm{kbps}$

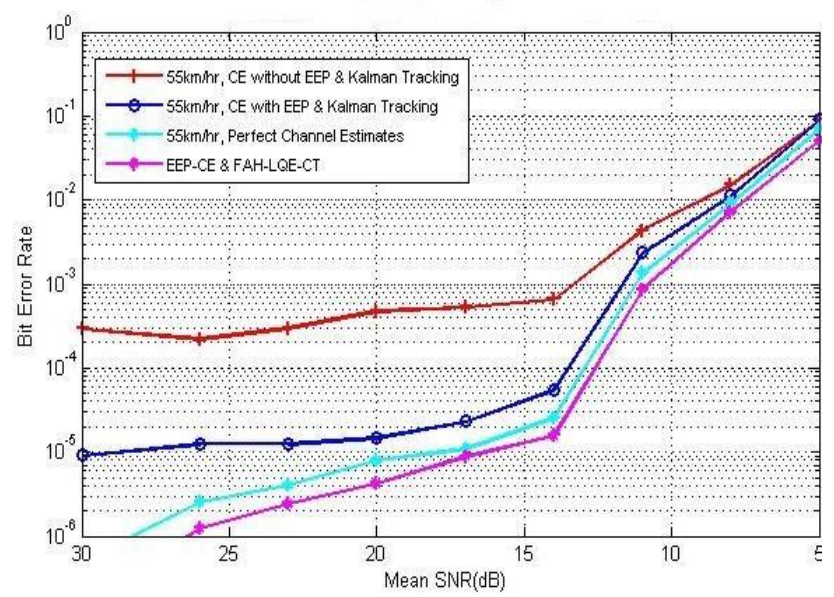

Figure. 8 BER performance of $(3 \times 2)$ MIMO-OFDM system with $\mu=55 \mathrm{~km} / \mathrm{hr}$ channels and $R=600 \mathrm{kbps}$

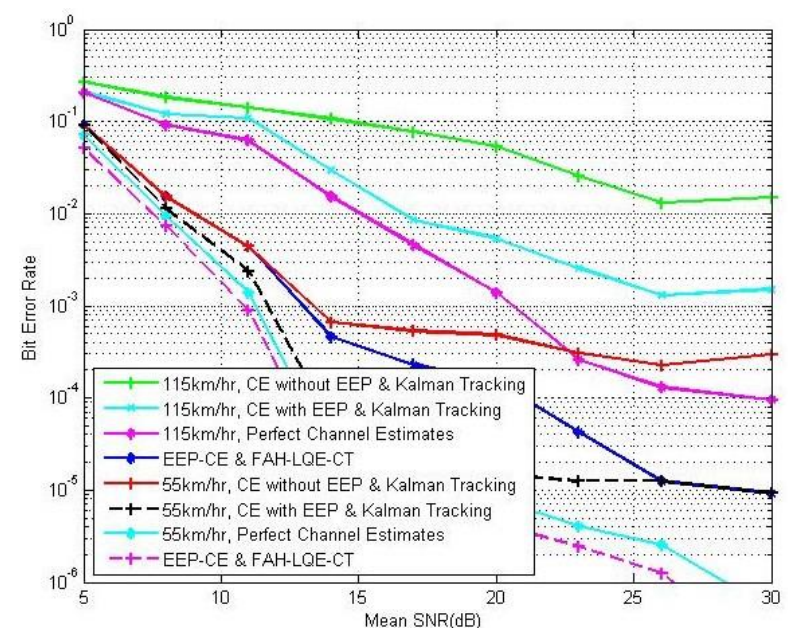

Figure. 9 Comparison analysis of BER performance for $(3 \times 2)$ MIMO-OFDM system with $\mu=115 \mathrm{~km}$. $/ \mathrm{hr}$ and $55 \mathrm{~km} / \mathrm{hr}$ channels and $R=600 \mathrm{kbps}$

The bit error rate (BER) system performance of a (3x2) MIMO-OFDM system under both TS and FS fading channels is shown in Fig. 9. The results are plotted for two mobile velocities for four different conditions: a) perfect $\mathrm{CE}$, b) $\mathrm{CE}$ with $\mathrm{EEP}$ \& Kalman-CT c) CE without EEP \& Kalman-CT and (d) FAH-LQE-CT. The results show the potential of the proposed EEP-CE and FAH-LQECT features in achieving a better symbol recovery and BER when utilized together, outperforming conventional $\mathrm{CE}$ and $\mathrm{CT}$.

\section{Conclusion}

This paper has presented a robust channel estimator with a hybrid EEP-CE and FAH-LQE-CT technique for MIMO-based systems that operate in both frequency and time selective fading channel. Extensive analysis of simulation has compared with conventional approaches proposed in the literature. The advantage of having our proposed technique is that the new jointly hybrid CE-EEP scheme can effectively predict the length of CIR and channel time-variation as well as carry out more robust channel estimation for MIMO systems. The FAHLQE channel tracking algorithm with decision feedback has also been incorporated to assist the hybrid CE-EEP process and cater for the case of TS channels. The functioning of proposed scheme has been corroborated and it is found that the proposed system is capable to provide effective and robust estimation of a channel in MIMO with potential tracking provision. The proposed methods yield a positive BER performance that is close to the results when perfect channel estimates and linear tracking are assumed. Comparative analysis with conventional $\mathrm{CE}$ algorithms presented in the literature also provided in the discussion, our proposed method reduced the BER at lower SNR values, which will enhance the system efficiency and robustness.

Furthermore, this can be implemented on various channel conditions and all the logics applied for estimating and tracking the channel should be tuned to dynamic nature.

\section{References}

[1] Y. Liu, Z. Tan, H. Hu, L. J. Cimini Jr., and G. Y. $\mathrm{Li}$, "Channel Estimation for OFDM", IEEE Communication Surveys \& Tutorials, Vol. 16, No. 4, Fourth Quarter 2014.

[2] D. Benyamina, A. Hafid, and M. Gendreau, "Wireless Mesh Networks Design - A Survey", Communications Surveys \& Tutorials, IEEE, Vol.14, No.2, pp.299-310, 2012.

[3] G. L. Stuber, J. R. Barry, S. W. McLaughlin, Y. Li, M. A. Ingram, and T.G. Pratt, "Broadband MIMO-OFDM Wireless Communications", In: 
Proc. of IEEE, Vol. 92, No. 2, pp. 271-294, 2004.

[4] Y.G. Li, J.H. Winters, and N.R. Sollenberger, "MIMO-OFDM for wireless communications: signal detection with enhanced channel estimation", IEEE Trans. Commun., Vol. 50, No. 9, pp. 1471-1477, 2002.

[5] S. E. Bensley and B. Aazhang, "Subspace-based channel estimation for code division multiple access communication systems", IEEE Trans. Commun., Vol. 44, No. 8, pp. 1009-1020, 1996.

[6] B. Muquet, M. De Courville, and P. Duhamel, "Subspace-based blind and semi-blind channel estimation for OFDM systems", IEEE Trans. Signal Process., Vol. 50, No. 7, pp. 1699-1712, 2002.

[7] R. O. Schmidt, "Multiple emitter location and signal parameter estimation", IEEE Trans. Antennas Propag., Vol. 34, No. 3, pp. 276-280, 1986.

[8] R. Roy and T. Kailath, "ESPRIT-estimation of signal parameters via rotational invariance techniques", IEEE Trans. Acous., Speech, Signal Process., Vol. 37, No. 7, pp. 984-995, 1989.

[9] B. Yang, "Projection approximation subspace tracking", IEEE Trans. Signal Process., Vol. 43, No. 1, pp. 95-107, 1995.

[10] P. Strobach, "Low-rank adaptive filters", IEEE Trans. Signal Process., Vol. 44, No. 12, pp. 2932-2947, 1996.

[11] A. GoldSmith, "Wireless Communication", Cambridge University Press, 2005.

[12] S. Rajasekaran and G. A. V. Pai, "Neural networks, fuzzy logic, and genetic algorithms", Prentice Hall of India, 2003.

[13] B. S. Chen, C. Y. Yang, and W. J. Liao, "Robust fast time-varying multipath fading channel estimation and equalization for MIMOOFDM systems via a fuzzy method", IEEE Transactions on Vehicular Technology, Vol. 64, No. 4, pp. 1599-1609, 2012.

[14] M. E. T Roman and V. Koivunen, "Timedomain method for tracking dispersive channels in mimo-ofdm systems", In: Proc. IEEE Intl. Conf. on Multimedia and Expo, Baltimore, MD, USA, pp. 393-396, 2003.

[15] Y. Li, "Simplified channel estimation for OFDM systems with multiple transmit antennas", IEEE Trans. Wireless Commun., Vol. 1, No. 1, pp. 67-75, 2002.

[16] B. Yang, K. Letaief, R. S. Cheng, and Z. Cao, "Channel estimation for OFDM transmission in multipath fading channels based on parametric channel modeling", IEEE Trans. Commun., Vol. 49, No. 3, pp. 467-479, 2001.

[17] I. Barhumi, G. Leus, and M. Moonen, "Optimal training design for MIMO OFDM systems in mobile wireless channels", IEEE Trans. Signal Process., Vol. 51, No. 6, pp. 1615-1624, 2003.

[18] H. Yin, D. Gesbert, M. Filippou, and Y. Liu, "A Coordinated Approach to Channel Estimation in Large-scale Multiple Antenna Systems", IEEE J. on Selected Areas in Communications, Vol. 31, No. 2, pp. 264-273, 2013.

[19] S.H. Huang, T. Jenho, T.C. Yang, and S.W. Cheng, "Model-Based Signal Subspace Channel Tracking for Correlated Underwater Acoustic Communication Channels", IEEE Journal of Oceanic Engineering, Vol.39, No.2, pp.343-356, 2014.

[20] K. Zhong, X. Lei, and S. Li, "Iterative channel estimation and data detection for MIMO-OFDM systems operating in time frequency dispersive channel under unknown background noise", EURASIP J. on Wireless Comms. Networking, 2013.

[21] H. Yin, D. Gesbert, M. Filippou, and Y. Liu, "A coordinated approach to channel estimation in large-scale multiple-antenna systems", IEEE J. Sel. Areas Commun., Vol. 31, No. 2, pp. 264 273, 2013.

[22] R. R. Muller, L. Cottatellucci, and M. Vehkapera, "Blind pilot decontamination", IEEE J. Sel. Topics Signal Process., Vol. 8, No. 5, pp. 773-786, 2014.

[23] H. Yin, L. Cottatellucci, D. Gesbert, R. R. Muller, and G. He, "Robust pilot decontamination based on joint angle and power domain discrimination", IEEE Trans. Signal Process., Vol. 64, No. 11, pp. 2990-3003, 2016.

[24] P. Dent, G. Bottomley, and T. Croft, "Jakes fading model revisited", Elec. Letters, Vol. 29, No. 13, pp. 1162-1163, 2006.

[25] Y. S. Hussein, M. Y. Alias, and A. A. Abdulkafi, "On Performance Analysis of LS and MMSE for Channel Estimation in VLC Systems", In: Proc. of the 12th International Colloquium on Signal Processing \& its Applications (CSPA2016), Malacca City, Malaysia, pp. 204-209, 2016.

[26] R. M. Prasad, "Navigate Symbol Assisted Channel Estimation Algorithms Under Various Channel Distribution", International Journal of Advanced Engineering Research and Science, Vol. 3, No. 9, pp. 172-178, 2016. 